\title{
The Sequential Price Of Anarchy for Atomic Congestion Games ${ }^{\star}$
}

\author{
Jasper de Jong and Marc Uetz \\ University of Twente, P.O. Box 217, 7500 AE Enschede, The Netherlands \\ $\{j \cdot$ dejong-3, m.uetz $\}$ Outwente.nl
}

\begin{abstract}
In situations without central coordination, the price of anarchy relates the quality of any Nash equilibrium to the quality of a global optimum. Instead of assuming that all players choose their actions simultaneously, here we consider games where players choose their actions sequentially. The sequential price of anarchy, recently introduced by Paes Leme, Syrgkanis, and Tardos [11], then relates the quality of any subgame perfect equilibrium to the quality of a global optimum. The effect of sequential decision making on the quality of equilibria, however, depends on the specific game under consideration. Here we analyze the sequential price of anarchy for atomic congestion games with affine cost functions. We derive several lower and upper bounds, showing that sequential decisions mitigate the worst case outcomes known for the classical price of anarchy $[5,2]$. Next to tight bounds on the sequential price of anarchy, a methodological contribution of our work is, among other things, a "factor revealing" integer linear programming approach that we use to solve the case of three players.
\end{abstract}

\section{Model and Notation}

We consider atomic congestion games with affine latency functions. The input of an instance $I \in \mathcal{I}$ consists of a finite set of resources $R$, a finite set of players $N=\{1, \ldots, n\}$, and for each player $i \in N$ a collection $\mathcal{A}_{i}$ of possible actions $A_{i} \subseteq R$. In other words, each players' action is to choose a subset of resources $A_{i}$ that are feasible for him. We say a resource $r \in R$ is chosen by player $i$ if $r \in A_{i}$, where $A_{i}$ is the action chosen by player $i$. By $A=\left(A_{i}\right)_{i \in N}$ we denote a possible outcome, that is, a complete profile of actions chosen by all players $i \in N$.

Each resource $r \in R$ has a constant activation cost $d_{r} \geq 0$ and a variable cost or weight $w_{r} \geq 0$ that expresses the fact that the resource gets more congested the more players choose it. The total cost of resource $r \in R$, for some outcome $A$, is then $f_{r}(A)=d_{r}+w_{r} \cdot n_{r}(A)$, where $n_{r}(A)$ denotes the number of players choosing resource $r$ in outcome $A$. Given outcome $A$, the negative utility of player $i$ is the total cost of all resources chosen by that player $\operatorname{cost}_{i}(A)=\sum_{r \in A_{i}} f_{r}(A)$. Players aim to minimize their costs. For later convenience, the total constant

\footnotetext{
* Research supported by CTIT (www.ctit.nl) and 3TU.AMI (www.3tu.nl), project "Mechanisms for Decentralized Service Systems".
} 
costs of a set of resources $T \subseteq R$ is denoted by $d(T)=\sum_{r \in T} d_{r}$, and the total weight of a set $T \subseteq R$ is denoted by $w(T)=\sum_{r \in T} w_{r}$. The total cost over all players of an outcome $A$ is denoted by $\operatorname{cost}(A)=\sum_{i \in N} \operatorname{cost}_{i}(A)$.

Note that this class of problems includes as a special case the celebrated network routing games as studied e.g. in [2,13], where the resources $R$ are network arcs, and actions for any player $i$ are origin-destination paths $A_{i}$ for that player. Another special case is singleton congestion games, where actions $A_{i}$ are all singletons, $\left|A_{i}\right|=1$. Here, resources $R$ can be thought of as machines or servers, with processing speeds $w_{r}^{-1}$, and each player selects, from a given subset of machines $\mathcal{A}_{i}$, a single machine to be processed on. This model, and variants thereof, are also known as load balancing games and, with respect to the quality of equilibria, have a vast literature, e.g. $[10,4,15]$.

Pure Nash equilibria are outcomes $\left(A_{i}\right)_{i \in N}$ in which no player can decrease her costs by unilaterally deviating from choosing $A_{i}$. The price of decentralization, better known as the price of anarchy $P o A$ [8], measures the quality of any Nash equilibrium relative to the quality of a globally optimal allocation, $O P T$. Here $O P T$ is an outcome minimizing the total costs over all players ${ }^{1}$. More specifically, for an instance $I$,

$$
P o A(I)=\max _{N E \in N E(I)} \frac{\operatorname{cost}(N E)}{\operatorname{cost}(O P T)},
$$

where $N E(I)$ denotes the set of all Nash equilibria for instance $I$. The price of anarchy of a class of instances $\mathcal{I}$ is defined by $P o A(\mathcal{I})=\sup _{I \in \mathcal{I}} P o A(I)$.

In this paper our goal is to compare the quality of Nash equilibria to the quality of subgame perfect equilibria of an extensive form game as introduced in $[9,14]$. We assume that the players choose their actions in an arbitrary, predefined order $1,2, \ldots, n$, so that the $i$-th player must choose his action $A_{i}$, observing the actions of players preceding $i$, but of course not knowing the actions of the players succeeding him. A strategy $S_{i}$ then specifies for player $i$ the actions he chooses, one for each potential profile of actions chosen by his predecessors $1, \ldots, i-1$. We denote by $S$ a strategy profile $\left(S_{i}\right)_{i \in N}$. The outcome $A=\left(A_{i}\right)_{i \in N}$ of a game is then the set of actions chosen by each player resulting from a given strategy profile $S$. Note that, if $S$ is a strategy profile of a subgame perfect equilibrium, the resulting outcome $A=\left(A_{i}\right)_{i \in N}$ is not necessarily a Nash equilibrium of the corresponding strategic form game; we will come back to this issue later in Section 4.1.

Extensive form games can be represented in a game tree, with the nodes on one level representing the possible situations that a single player can encounter, and the edges emanating from any node representing the possible actions of that player in the given situation. The nodes of the game tree are also called infor-

\footnotetext{
${ }^{1}$ Note that we consider a utilitarian global objective, that is, the global objective is to minimize the sum of the costs of all players. This is one of the standard models, yet different than the egalitarian makespan objective as studied, e.g., in [8].
} 
mation sets ${ }^{2}$. See Figure 2 for an example. Subgame perfect equilibria, defined by Selten [14], are defined as strategy profiles that induce Nash equilibria in any subgame of the game tree. Analogous to (1), the sequential price of anarchy of an instance $I$ is defined by

$$
S P o A(I)=\max _{S P E \in S P E(I)} \frac{\operatorname{cost}(S P E)}{\operatorname{cost}(O P T)},
$$

where $S P E(I)$ denotes the set of all subgame perfect equilibria of instance $I$ in extensive game form. The sequential price of anarchy of a class of instances $\mathcal{I}$ is defined as in [11] by $S P o A(\mathcal{I})=\sup _{I \in \mathcal{I}} S P o A(I)$. Throughout the paper, when the class of instances is clear from the context, we write $P o A$ and $S P O A$.

\section{Related Work and Contribution}

Recently, the sequential price of anarchy was introduced by Paes Leme et al. [11] as an alternative way to measure of the costs of decentralization. Compared to the classical price of anarchy of Papadimitriou and Koutsoupias [8], it avoids the "curse of simultaneity" inherent in certain games [11]. More specifically, for machine cost sharing games, generic unrelated machine scheduling games and generic consensus games, the $S P o A$ is smaller than the $P o A$ [11]. However, for the latter two games, the 'generic' condition is indeed necessary [3]. Moreover, Bilò et al. [3] show that for many games myopic behaviour leads to better equilibria than the farsighted behaviour of subgame perfect equilibria. Also for throughput scheduling games, or more generally, set packing games, the $S P O A$ is lower than the $P o A$ [6]. For isolation games, however, the $S P o A$ is worse than the $P o A$ in general [1]. These results leave a mixed impression, and lead to the natural question which classes of games possess a $S P O A$ which is lower than the $P o A$. We give a (partial) answer to this question for atomic congestion games with affine cost functions. Congestion games have been introduced by Rosenthal [12]. For this class of games, the price of anarchy is known to equal 2 in the case of two players, and 2.5 in the case of three or more players [5,2].

Our contributions are both lower and upper bounds on the sequential price of anarchy for atomic congestion games with affine cost functions. For two players, we prove a tight bound of 1.5 . For three players, we use integer linear programming to obtain a tight bound of $2 \frac{63}{488} \approx 2.13$. This bound is obtained by first using simple combinatorial arguments to show that the worst case must be attained by some instance that is moderate in size, and then computing this worst case instance with a standard ILP solver. For $n>3$ players, we were not (yet) able to prove a constant upper bound, which we conjecture to be smaller than 2.5. We know that $2 \frac{63}{488} \leq S P o A \leq n$, and when the number of players increases, we give a parametric family of instances that yields a lower bound of $2+1 / e \approx 2.37$ on the $S P o A$. We also consider the special case of singleton congestion games

\footnotetext{
${ }^{2}$ We deal with a game with perfect information, so all information sets are trivial, and subgame perfect equilibria correspond to backward induction equilibria.
} 
for which the $P o A$ is 2.5 [4]. Here, the parametric lower bound example with $S P O A=2+1 / e$ holds, and we find an upper bound slightly smaller that trivial, namely $n-1$. The latter is interesting mainly for the case of $n=3$ players. We substantially improve on these results for symmetric singleton congestion games, where we show that the $S P O A$ equals $4 / 3$, which matches the bound known for the $P o A$ [7]. Along the way, we present some additional insights.

\section{An Illustrative Example}

Example 1. There are two players $N=\{1,2\}$, and three resources $R=\{1,2,3\}$ with zero constant costs and weights $w_{1}=w_{2}=1, w_{3}=2$. Player 1 can choose either resource 1 or resource 2. Player 2 can choose either resource 2 or resource 3. This example is shown in Figure 1.

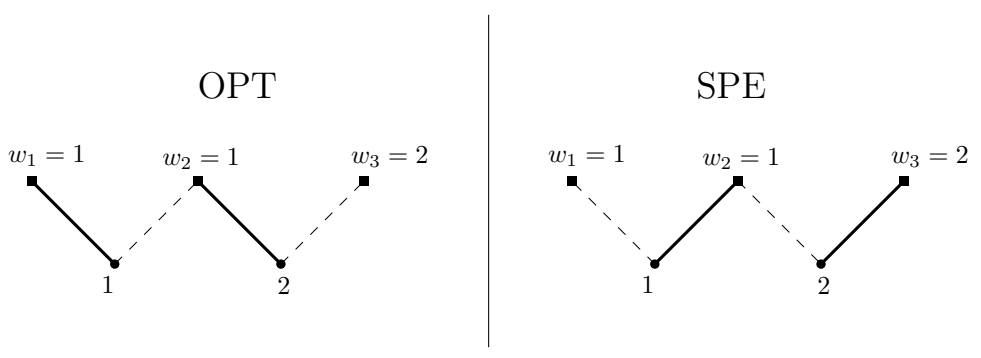

Fig. 1. A simple example. Dots represent to players, squares represent resources, and edges represent actions. Fat edges correspond to chosen actions

It is clearly optimal when player 1 chooses resource 1 , and player 2 chooses resource 2 , since in that case, both players choose their action of minimum weight and the resources do not overlap. However, in the worst case subgame perfect equilibrium, player 1 chooses resource 2 , and player 2 chooses resource 3 . Note that player 2 chooses resource 3 , since his total costs are the same as when he would choose resource 2 . Also note that player 1 chooses resource 2 due to his farsighted behaviour; he knows player 2 will not choose resource 2 in this equilibrium. The latter observation might seem counterintuitive, especially since choosing resource 2 is a weakly dominated strategy for player 1 in the corresponding strategic form game. However, we can easily convert all lower bound examples in this paper to examples with unique (and strict) equilibria. For this example we could decrease the weight of resource 2 by some small constant $\epsilon$, and decrease the weight of resource 3 by $3 \epsilon$.

We see from this example that $S P o A \geq \frac{1+2}{1+1}=1.5$. It turns out that this simple example yields a tight bound, as we show in the next section. 


\section{The Sequential Price of Anarchy for Two Players}

We now prove that $S P o A \leq 1.5$, which along with the observation of Section 3 shows that the sequential price of anarchy for atomic congestion games with linear latency functions for 2 players is exactly 1.5 .

We need the following notation: Denote by $O P T=\left(A_{11}, A_{21}\right)$ the pair of actions of two players 1 and 2, respectively, in an optimal allocation. Denote by $S P E=\left(A_{12},\left(A_{22}, A_{23}\right)\right)$ the actions in any subgame perfect equilibrium. Here, $A_{11}, A_{12} \in \mathcal{A}_{1}$, and $A_{21}, A_{22}, A_{23} \in \mathcal{A}_{2}$. This notation should be understood as follows: In $S P E$ player 1 prefers to choose $A_{12}$. If player 1 were to choose $A_{11}$, then it is subgame perfect for player 2 to choose $A_{22}$. If player 1 were to choose $A_{12}$, then it is subgame perfect for player 2 to choose $A_{23}$. Note that both players might have more actions at their disposal, however these are not relevant for the analysis. It suffices to consider two actions for the first player, one for the optimal allocation, $A_{11}$, and one for the subgame perfect equilibrium, $A_{12}$. For the second player, it suffices to consider the action for the optimal allocation, $A_{21}$, plus the actions that player 2 were to play in reaction to $A_{11}$ and $A_{12}$, which are $A_{22}$ and $A_{23}$, respectively. Also note that we do not exclude cases where any two sets from $A_{11}, A_{12}, A_{21}, A_{22}$ or $A_{23}$ overlap. It could even be that they are equal. The general situation is shown in Figure 2.

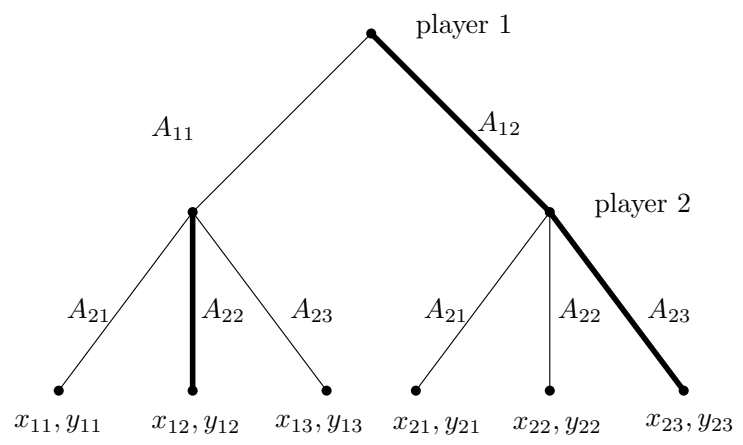

Fig. 2. All relevant actions in the game tree for 2 players. Values at the leaves denote the costs for both players. Fat lines correspond to subgame perfect actions.

For ease of notation, let $a=d\left(A_{11}\right), b=d\left(A_{21}\right), \alpha=w\left(A_{11} \backslash A_{21}\right), \beta=$ $w\left(A_{21} \backslash A_{11}\right), \gamma=w\left(A_{11} \cap A_{21}\right), \delta=w\left(A_{11} \cap A_{22}\right)-w\left(A_{11} \cap A_{21}\right)$. So $\delta$ denotes the difference in the total weight of shared resources, when player 1 chooses $A_{11}$ and player 2 switches from $A_{21}$ to $A_{22}$. Let $x_{a b}=\operatorname{cost}_{1}\left(A_{1 a} A_{2 b}\right)$ and let $y_{a b}=\operatorname{cost}_{2}\left(A_{1 a} A_{2 b}\right)$. Then, $\operatorname{cost}(O P T)=x_{11}+y_{11}=a+\alpha+b+\beta+4 \gamma$. Since player 2 prefers $y_{12}$ to $y_{11}$, we get $y_{12} \leq y_{11}=b+\beta-2 \delta+2(\gamma+\delta)$. We prove the theorem by upper bounding $\operatorname{cost}(S P E)$, by deriving an upper bound on $x_{23}$ (Lemma 1), and two upper bounds on $y_{23}$ (Lemmas 2 and 3). 
Lemma 1. $x_{23} \leq a+\alpha+2 \gamma+\delta$

Proof. $x_{23} \leq x_{12}=d\left(A_{11}\right)+w\left(A_{11}\right)+w\left(A_{11} \cap A_{22}\right)=a+(\alpha+\gamma)+(\gamma+\delta)=$ $a+\alpha+2 \gamma+\delta$. The inequality follows from the fact that player 1 prefers $x_{23}$ to $x_{12}$. The first equality follows from the definition of $x_{12}$; it denotes the costs for player 1 when player 1 chooses $A_{11}$ and player 2 chooses $A_{22}$.

Lemma 2. $y_{23} \leq 2(b+\beta+\gamma-\delta)$

Proof. $y_{23} \leq y_{22} \leq d\left(A_{22}\right)+2 w\left(A_{22}\right) \leq 2\left(d\left(A_{22}\right)+w\left(A_{22}\right)\right)$. The first inequality holds since player 2 prefers $y_{23}$ to $y_{22}$. The second inequality follows from the fact that each resource can be chosen by at most two players. We now show that $d\left(A_{22}\right)+w\left(A_{22}\right) \leq b+\beta+\gamma-\delta$, proving the lemma. Since player 2 prefers $y_{12}$ to $y_{11}$, we get $y_{12} \leq y_{11}=b+\beta+2 \gamma=b+\beta-2 \delta+2(\gamma+\delta)$. Since $y_{12}=d\left(A_{22}\right)+w\left(A_{22} \backslash A_{11}\right)+2 w\left(A_{11} \cap A_{22}\right)$ and $w\left(A_{11} \cap A_{22}\right)=\gamma+\delta$, we get $d\left(A_{22}\right)+w\left(A_{22} \backslash A_{11}\right) \leq b+\beta-2 \delta$. Therefore $d\left(A_{22}\right)+w\left(A_{22}\right)=$ $d\left(A_{22}\right)+w\left(A_{11} \cap A_{22}\right)+w\left(A_{22} \backslash A_{11}\right) \leq b+\beta+\gamma-\delta$.

Lemma 3. $y_{23} \leq a+\alpha+b+\beta+3 \gamma$

Proof. $y_{23} \leq y_{22} \leq d\left(A_{22}\right)+w\left(A_{22}\right)+w\left(A_{12}\right)$. The first inequality holds since player 2 prefers $y_{23}$ to $y_{22}$. The second inequality follows from the fact that each resource that player 1 chooses adds at most the weight of that resource to the cost of player 2. We know from the proof of Lemma 2 that $d\left(A_{22}\right)+w\left(A_{22}\right) \leq$ $b+\beta+\gamma-\delta$. We know from the proof of Lemma 1 that $x_{12} \leq a+\alpha+2 \gamma+\delta$. Player 1 could secure himself a total cost of $x_{12}$ by choosing $A_{11}$. Since he chooses $A_{12}$ in $S P E, w\left(A_{12}\right) \leq x_{12} \leq a+\alpha+2 \gamma+\delta$. Now $d\left(A_{22}\right)+w\left(A_{22}\right)+w\left(A_{12}\right) \leq$ $b+\beta+\gamma-\delta+a+\alpha+2 \gamma+\delta=a+\alpha+b+\beta+3 \gamma$.

Theorem 1. SPoA $=1.5$ for atomic congestion games with two players and affine cost functions.

Proof. Recall, $\operatorname{cost}(O P T)=x_{11}+y_{11}$ and $\operatorname{cost}(S P E)=x_{23}+y_{23}$. Using Lemmas 1,2 and 3 , we get $2\left(x_{23}+y_{23}\right) \leq 2 a+2 \alpha+2(2 \gamma+\delta)+2(b+\beta+\gamma-\delta)+a+\alpha+b+$ $\beta+3 \gamma=3 a+3 \alpha+3 b+3 \beta+9 \gamma \leq\left(x_{11}+y_{11}\right) \frac{3 a+3 \alpha+3 b+3 \beta+9 \gamma}{a+\alpha+b+\beta+4 \gamma} \leq 3\left(x_{11}+y_{11}\right)$.

\subsection{Reflection on Proof Techniques}

The above proof is quite lengthy, especially considering the simplicity of our lower bound example. However, contrary to what one might expect, not every outcome of a subgame perfect equilibrium is a Nash equilibrium of the corresponding strategic form game ${ }^{3}$. Also, as with Nash equilibria, it is possible that both players have a higher cost than in the optimum. These two effects are illustrated in the following example.

\footnotetext{
${ }^{3}$ Note that both games have different strategy spaces: In the strategic form game both players have as strategy space their feasible actions, $\mathcal{A}_{i}$. In the extensive form game, however, the strategy space for the second player is more-dimensional, as it specifies an action $A_{2} \in \mathcal{A}_{2}$ for all information sets (= possible actions of player 1 ).
} 
Example 2. There are two players $N=\{1,2\}$, and four resources $R=\{1,2,3,4\}$ with zero constant costs $d_{r}=0, \forall r \in R$ and weights $w_{1}=7, w_{2}=4, w_{3}=$ $1, w_{4}=19$. Player 1 can choose either resource 1 or resources $\{1,2,3\}$. Player 2 can choose either resources $\{1,2\}$ or resources $\{3,4\}$. This example is shown in Figure 3.

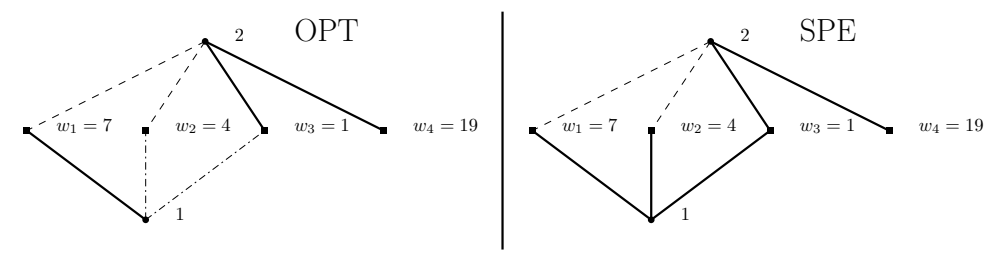

Fig. 3. In this example the $S P E$ is bad for both players. Dots represent players, and squares represent resources. Fat lines correspond to chosen resources. Note that lines do not represent actions, only resources that can be chosen.

In the social optimum, player 1 chooses resource 1 , and player 2 chooses resources $\{3,4\}$, which yields a total cost of $7+(1+19)=27$. However, if player 1 were to choose resource 1 , then it is subgame perfect for player 2 to choose $\{1,2\}$, which yields him a cost of $7 \cdot 2+4=18 \leq 19+1=20$. This yields player 1 a cost of $7 \cdot 2=14$. Therefore it is subgame perfect for player 1 to choose $\{1,2,3\}$, since then it is subgame perfect for player 2 to choose $\{3,4\}$, which yields him a cost of $19+2 \cdot 1=21 \leq 2 \cdot 7+2 \cdot 4=22$. In this equilibrium, player 1 has cost $7+4+2 \cdot 1=13$. Note that both players have higher cost in SPE than in $O P T$. Also, the subgame perfect equilibrium is not a Nash equilibrium of the corresponding strategic form game, since player 1 plays an action that is strictly dominated in the strategic form game.

This example shows that well known proof techniques, like smoothness or potential arguments are problematic in a sequential setting; the arguments typically rely on the fact that in a Nash equilibrium outcome, no player is willing to deviate.

\section{The Sequential Price of Anarchy for Three Players}

Along the lines of the proof for the case with two players, we also settle the case with three players. To that end, we use an integer linear programming (ILP) approach. We first use simple combinatorial arguments to argue that a worst case instance is moderate in size. This is done in Lemmas 4,5 , and 6 . We then simply compute a worst case instance using a standard ILP solver. To prove the lemmas, we use the following notation: Define the series

$$
x_{1}:=2 \text { and } x_{i}:=1+\prod_{j \prec i} x_{i} \text { for all } i \geq 2 .
$$


Note that $x_{2}=3, x_{3}=7, x_{4}=43$, and that $x_{i}$ grows super-exponentially.

Lemma 4. For any instance $I$, there exists an instance $I^{\prime}$ with $\left|\mathcal{A}_{i}\right| \leq x_{i}$ for all players $i \in N$ for which $S P o A\left(I^{\prime}\right)=S P o A(I)$.

Proof. The proof goes by successively eliminating all actions that are not played in a fixed worst case $S P E$ from $I$, in the order of the players $1,2, \ldots, n$. For the first player, we thereby reduce $\mathcal{A}_{1}$ to only two actions, one in $O P T$ and one in a worst case $S P E$. For the second player, we thereby restrict $\mathcal{A}_{2}$ to $x_{2} \leq 3$ actions, the $S P E$ actions in two information sets, one for each possible action of the first player, plus the action in $O P T$. More generally, for the $k$ th player, we reduce $\mathcal{A}_{k}$ to at most $1+\prod_{i \prec k} x_{i}$ actions, namely the subgame perfect actions of the fixed $S P E$ in each of the at most $\prod_{i \prec k} x_{i}$ information sets, plus the action in $O P T$. In the so reduced instance, the $S P E$ that we started with is still subgame perfect, as the actions that were removed are all actions with inferior or identical outcome for the respective player.

Lemma 5. For any instance $I$, there exists an instance $I^{\prime}$ with $\left|\mathcal{A}_{i}\right| \leq x_{i}$ for all players $i \in N$, and any two resources are not part of the exact same set of actions, and hence, $|R| \leq 2^{\sum_{i \in N}\left|\mathcal{A}_{i}\right|}$, and $S P o A\left(I^{\prime}\right)=S P o A(I)$.

Proof. By Lemma 4, we may restrict w.l.o.g. to instances $I$ with $\left|\mathcal{A}_{i}\right| \leq x_{i}$ for all players $i$. Suppose the claim is false. Then choose among all instances $I$ that falsify the claim an instance with minimal set of resources $R$. Let $r$ and $r^{\prime}$ be two resources that are part of the exact same set of actions. We construct an instance $I^{\prime}$ which is a identical to $I$, except instead of $r$ and $r^{\prime}$, it contains a resource $r^{\prime \prime}$ for which $w_{r^{\prime \prime}}=w_{r}+w_{r^{\prime}}$ and $d_{r^{\prime \prime}}=d_{r}+d_{r^{\prime}}$. Now note that $I^{\prime}$ has the same sets of actions as $I$. Now each outcome $\left(A_{i}\right)_{i \in N}$ in $I^{\prime}$ has the same costs as $\left(A_{i}\right)_{i \in N}$ in $I$. Therefore the same actions are subgame perfect and $S P o A\left(I^{\prime}\right)=S P o A(I)$. As $I^{\prime}$ has one resource less, we obtain a contradiction. Finally, due to the fact that no two resources are part of the exact same set of actions, and since there are in total no more than $\sum_{i \in N}\left|\mathcal{A}_{i}\right|$ actions, by the pigeonhole principle there cannot be more than $2^{\sum_{i \in N}\left|\mathcal{A}_{i}\right|}$ resources.

Lemma 6. For any instance $I$, there exists an instance $I^{\prime}$ with $\left|\mathcal{A}_{i}\right| \leq x_{i}$ for all players $i \in N$, any 2 resources are not part of the exact same set of actions, $|R| \leq 2^{\sum_{i \in N}\left|\mathcal{A}_{i}\right|}$ and $d_{r}+w_{r} \leq n \operatorname{cost}(O P T)$ for all resources $r \in R$, such that $\operatorname{SPoA}\left(I^{\prime}\right)=\operatorname{SPoA}(I)$.

Proof. The first three claims follow by the two previous lemmas. Next, observe that resources $r$ with $d_{r}+w_{r}>n \operatorname{cost}(O P T)$ can safely be eliminated, as it cannot be subgame perfect for any player $i$ to choose resource $r$ : choosing $O P T_{i}$ instead, the action that player $i$ chooses in the optimal allocation, yields a cost at most $n \operatorname{cost}\left(O P T_{i}\right) \leq n \operatorname{cost}(O P T)<d_{r}+w_{r}$.

Specifically, for congestion games with three players, in order to find a worstcase instance we only need to consider instances of moderate size. It suffices to let $\mathcal{A}_{1}=2, \mathcal{A}_{2}=3, \mathcal{A}_{3}=7,|R|=2^{2+3+7}$, any 2 resources are not in the 
exact same set of actions and $d_{r}+w_{r} \leq 3 \operatorname{cost}(O P T)$ for all resources $r \in R$. Intuitively, the ILP works as follows: It maximizes the $S P o A$ over all instances with the properties described above. Let $\mathcal{A}=\bigcup_{i \in N} \mathcal{A}_{i}$ denote the set of all 12 actions. We have $2^{12}=4096$ resources, one for every possible combination of actions. The ILP decides the weight $w_{r}$ and constant cost $d_{r}$ of each resource. We define some fixed outcome as the social optimum with total cost normalized to 1, and maximize the $S P E$.

The details of the ILP are given in the appendix, section A. We have implemented this integer linear program using the AIMMS modeling framework, and using CPLEX 12.5 we obtain an optimal solution with value $2 \frac{63}{488}$. In that solution, player 3 only uses 4 different actions. The resulting instance (scaled to integer values) is given in the appendix, section $\mathrm{B}$, where we omit all trivial actions and resources. We conclude this section with the following.

Theorem 2. $S P o A=2 \frac{63}{488} \approx 2.13$ for atomic congestion games with three players and affine cost functions.

This result is particularly interesting in comparison to the tight bound 2.5 for the price of anarchy for (non-sequential) three player congestion games [5, 2].

\section{Results for $n>3$ players}

Given the techniques used so far, problems with $n>3$ players become increasingly difficult. Due to the the specific value $2 \frac{63}{488}$ for 3 players, it seems unlikely that we can find a simple, general bound using combinatorial arguments. Extending the ILP straightforwardly to the case with 4 players is also problematic; using Lemma 6 , we'd need to consider $2 \cdot 3 \cdot 7+1=43$ actions for player 4 , and $2^{55}$ resources. However, it is not unexpected that most of the gigantic amount of variables remain nonbasic (for three players we only need $2+3+4$ actions and 13 resources), which leaves ample room for future computational work and progress, e.g., using column generation. While we leave this for future work, here we present results for the special case of singleton congestion games.

\subsection{A Lower Bound for Singleton Congestion Games}

Here we construct a class of parametric lower bound examples $G(a, b)$, for integers $a, b \geq 0$, to prove a lower bound on the sequential price of anarchy for singleton congestion games that approaches $2+1 / e$ as the number of players increases.

Theorem 3. Asymptotically for $n \rightarrow \infty, S P o A \geq 2+\frac{1}{e} \approx 2.37$ for singleton atomic congestion games with linear cost functions.

The proof is by the following parametric set of instances. 
Example 3. The set of players $N$ is partitioned into subsets $N_{1}, \ldots, N_{a}$, $N_{a+1}, \ldots, N_{a+b}$. Any subset $N_{j}, j \in\{1, \ldots, a\}$ consists of $b$ ! players. Any subset $N_{j}, j \in\{a+1, \ldots, a+b\}$ consists of $\frac{b !}{(j-a) !}$ players. The set of resources $R,|R|=$ $n+1$ is partitioned into subsets $R_{1}, \ldots, R_{a}, R_{a+1}, \ldots, R_{a+b+1}$. Subset $R_{1}$ consists of $b$ ! resources of weight $2^{-a+1}$, any subset $R_{j}, j \in\{2, \ldots, a\}$ consists of $b$ ! resources of weight $2^{j-a-1}$ (note that these weights are between 0 and 1 ), any subset $R_{j}, j \in\{a+1, \ldots, a+b\}$ consists of $\frac{b !}{(j-a) !}$ resources of weight 1 , finally $R_{a+b+1}$ consists of a single resource of weight $b+1$. Any player from $N_{j}, j \in$ $\{1, \ldots, a+b\}$ can choose from exactly 2 sets, each consisting of one resource; one is a resource in $R_{j}$, and one is a resource in $R_{j+1}$. Each resource in $R_{1}$ can be chosen only by one player in $N_{1}$. Each resource in $R_{j}, j \in\{2, \ldots, a\}$ can be chosen by one player in $N_{j}$ and one player in $N_{j-1}$. Each resource in $R_{j}, j \in\{a+1, \ldots, a+b\}$ can be chosen by exactly $j-a+1$ players; 1 from $N_{j}$, and $j-a$ from $N_{j-1}$. Finally, the only resource in $R_{a+b+1}$ can be chosen only by the single player in $N_{a+b}$. Players are ordered by the number of their subset; players in $N_{1}$ choose first, then players in $N_{2}$, etc. The order of players within subsets is irrelevant. This example is shown in Figure 4, for $a=3, b=3$.

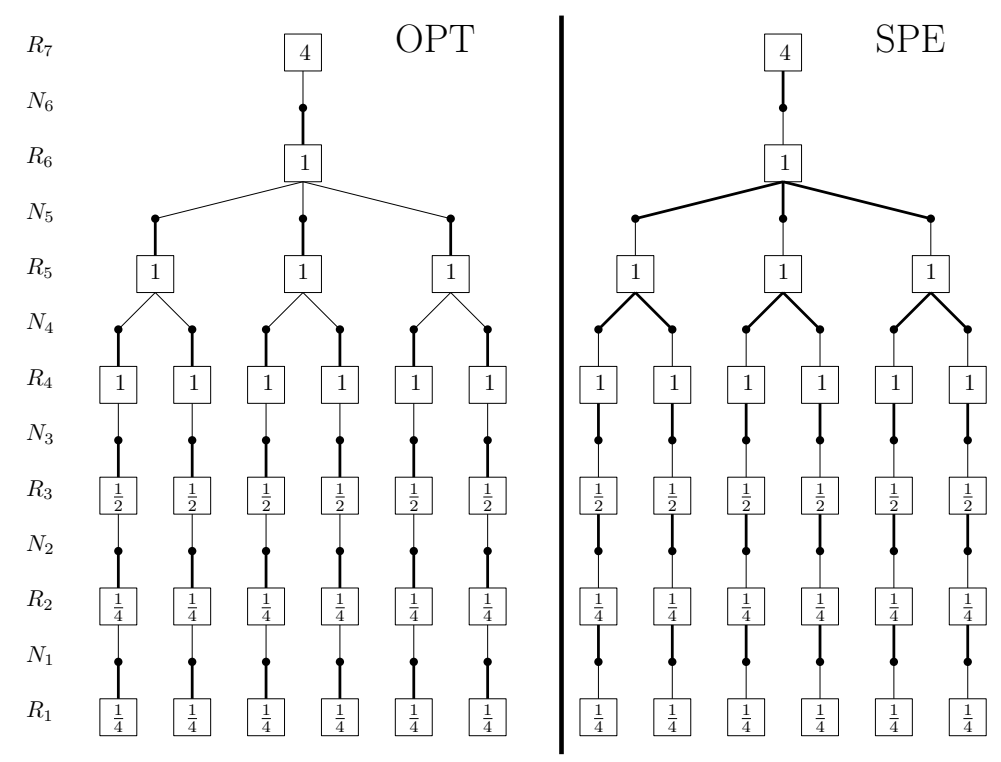

Fig. 4. This example shows that the $S P O A$ is at least $2+1 / e$ (asymptotically). Players are denoted by dots, and resources by squares. The number in each resource denotes its weight $w_{r}$, and fat lines indicate the actions. Here, constant costs $d_{r}=0$

It is easy to see that the social optimum is obtained when each player in $N_{j}, j \in$ $\{1, \ldots, a+b\}$ chooses a resource in $R_{j}$, since then each player chooses the resource 
with the lowest cost, and no resource is chosen by more than one player. This yields a total cost of $\sum_{j=1}^{b}\left(\frac{b !}{j !}\right)+\left(\sum_{j=2}^{a}\left(2^{j-a-1}\right)+2^{-a+1}\right) b !=\sum_{j=1}^{b}\left(\frac{b !}{j !}\right)+b !$. However, it is a subgame perfect equilibrium when each player in $N_{j}, j \in\{0, \ldots, a+b\}$ chooses a resource in $R_{j+1}$; each player in $N_{1}$ has a cost of $2^{-a+1}$, while by deviating, he has a cost of $2^{-a+1}$ as well. Each player in $N_{j}, j \in\{2, \ldots, a\}$ has a cost of $2^{j-a}$, while by deviating, he has a cost of $2^{j-a-1} \cdot 2=2^{j-a}$ as well. Each player in $N_{j}, j \in\{a+1, \ldots, a+b-1\}$ has a cost of $1(j-a+1)$, while by deviating, he has a cost of $1(j-a+1)$ as well. Finally, the only player in $N_{a+b}$ has a cost of $(b+1) 1$, while by deviating, he has a cost of $1(b+1)$ as well. This yields a total cost of $\sum_{j=1}^{b}\left(\frac{b !}{j !}(j+1)\right)+\sum_{j=1}^{a}\left(2^{-j+1} b !\right)=\sum_{j=1}^{b}\left(\frac{b !}{j !}(j+1)\right)+b !\left(2-2^{-a+1}\right)$. We see that

$$
S P o A \geq \frac{\sum_{j=1}^{b} \frac{b !}{j !}(j+1)+b !\left(2-2^{-a+1}\right)}{\sum_{j=1}^{b} \frac{b !}{j !}+b !} .
$$

Letting $a \rightarrow \infty$ we obtain

$$
S P o A \geq \frac{\sum_{j=1}^{b} \frac{b !}{j !}(j+1)+2 b !}{\sum_{j=1}^{b} \frac{b !}{j !}+b !} .
$$

Finally for $b \rightarrow \infty$, we conclude

$$
\lim _{b \rightarrow \infty} \frac{\sum_{j=1}^{b} \frac{b !}{j !}(j+1)+2 b !}{\sum_{j=1}^{b} \frac{b !}{j !}+b !}=\lim _{b \rightarrow \infty} \frac{\sum_{j=1}^{b} \frac{j+1}{j !}+2}{\sum_{j=1}^{b} \frac{1}{j !}+1}=\lim _{b \rightarrow \infty} \frac{\sum_{j=0}^{b} \frac{j+1}{j !}+1}{\sum_{j=0}^{b} \frac{1}{j !}},
$$

which equals $\frac{2 e+1}{e}$ by standard calculus. Therefore $S P O A \geq 2+\frac{1}{e} \approx 2.37$.

\subsection{An Upper Bound for Singleton Congestion Games}

For the special case of singleton congestion games, we obtain the following.

Theorem 4. For singleton atomic congestion games with affine cost functions, $S P O A \leq n-1$.

Proof. The proof is by contradiction. See the appendix, Section C.

Note that this is not much tighter than the trivial upper bound $n$ that already holds for general congestion games. It gives a small improvement, though, for the case of $n=3$ players over the upper bound $2 \frac{63}{488}$.

\subsection{Singleton Congestion Games with Symmetric Players}

Here we prove the following theorem for the special case of singleton, symmetric congestion games.

Theorem 5. For symmetric singleton atomic congestion games with affine cost functions, $S P O A=4 / 3$. 
To prove the theorem, we first derive a more general result

Theorem 6. For symmetric singleton atomic congestion games with non-decreasing latency functions, every subgame perfect equilibrium of the extensive form game is a Nash equilibrium of the corresponding strategic form game ${ }^{4}$.

Proof. Intuitively our proof is as follows: we show that for any player $i$ for which there exists a resource $r^{\prime}$ in the SPE outcome that is less costly than the resource $r$ that he chose, we can find a successor $j$ for which there also exists a less expensive resource in the SPE outcome in the subgame where player $i$ chooses $r^{\prime}$. With this, we construct a contradiction. Let us call a player ex-post discontent if, in the SPE outcome, there exists such a less expensive resource for that player. For simplicity of notation, let us assume that the set of all instances contains all subgames as well.

Among all instances that would falsify the claim, choose an instance $I$ which, among all such instances has the smallest number of players succeeding the last ex-post discontent player, $i$. Denote by $A$ the outcome of the SPE in that instance. Let $r$ be the resource that player $i$ chooses in the $S P E$, and let $r^{\prime}$ be the ex-post more attractive resource for player $i$. Denote by $A^{\prime}$ the resulting outcome when only player $i$ deviates ex-post (note that this is not an equilibrium). Denote by $A^{\prime \prime}$ the outcome of the $S P E$ of the subgame where players preceding $i$ choose the same actions as in $A$, and player $i$ chooses $r^{\prime}$ (again, this is not an equlibrium, but it is subgame perfect for the corresponding subgame for players succeeding i). Figure 5 may help to illustrate this.

We argue that in $A^{\prime \prime}$ at least one successor $j$ of $i$ chooses $r^{\prime}$ as well, since $f_{r^{\prime}}\left(A^{\prime}\right)<f_{r}(A) \leq f_{r^{\prime}}\left(A^{\prime \prime}\right)$. The first inequality follows from the fact that player $i$ improves in the strategic form game by choosing $r^{\prime}$ over $r$. The second inequality follows from the fact that it is subgame perfect for player $i$ to choose $r$ in the extensive form game. Therefore we may conclude that $n_{r^{\prime}}(A)<n_{r^{\prime}}\left(A^{\prime \prime}\right)$.

Denote by $T$ the set of resources chosen in $A$ by players succeeding $i$. For any resource $r^{\prime \prime} \in T$ we have $f_{r^{\prime \prime}}(A)<f_{r}(A)$, since otherwise some successor $j$ of $i$ can decrease his cost by deviating to $r^{\prime}$ in the strategic form game, contradicting the fact that $i$ has the fewest successors among all ex-post discontent players. We now compare outcomes $A$ and $A^{\prime \prime}$. Since $n_{r^{\prime}}(A)<n_{r^{\prime}}\left(A^{\prime \prime}\right)$, and all predecessors of $i$ choose the same resource in $A$ as in $A^{\prime \prime}$, there exists a resource $r^{\prime \prime} \in T$ for which $n_{r^{\prime \prime}}(A)>n_{r^{\prime \prime}}\left(A^{\prime \prime}\right)$. Now suppose that player $j$ deviates ex-post and chooses $r^{\prime \prime}$ instead of $r^{\prime}$. Denote the resulting outcome by $A^{\prime \prime \prime}$. We get $n_{r^{\prime \prime}}\left(A^{\prime \prime \prime}\right)=$ $n_{r^{\prime \prime}}\left(A^{\prime \prime}\right)+1 \leq n_{r^{\prime \prime}}(A)$, therefore $f_{r^{\prime \prime}}\left(A^{\prime \prime \prime}\right) \leq f_{r^{\prime \prime}}(A)$ which yields $f_{r^{\prime \prime}}\left(A^{\prime \prime \prime}\right) \leq$ $f_{r^{\prime \prime}}(A)<f_{r}(A) \leq f_{r^{\prime}}\left(A^{\prime \prime}\right)$. We see that ex-post deviating decreases the cost of player $j$. Since $j$ is a successor of $i$, this contradicts the fact that $i$ has the fewest successors among all ex-post discontent players. This contradicts the choice of $I$.

\footnotetext{
${ }^{4}$ What we mean here is the non-sequential strategic form game where the strategies of all players are just single actions.
} 


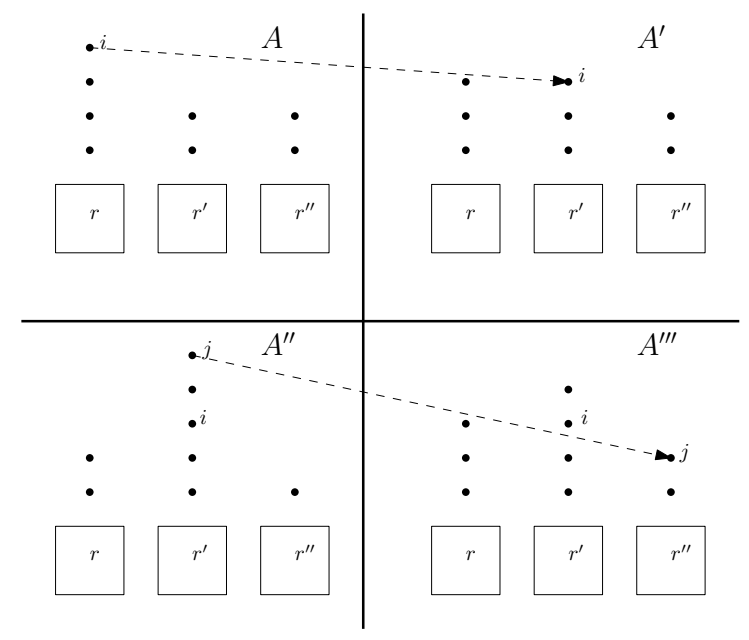

Fig. 5. This example depicts the 4 outcomes used in theorem 5. Dots represent players, and squares represent resources. When a player is positioned above a resource, that player chooses that resource.

Proof (for Theorem 5). The upper bound follows from Theorem 6, and the fact that $P o A=4 / 3$, which follows from [7]. The following lower bound example shows that this bound is tight. There are 2 players and 2 resources, $w_{1}=1, w_{2}=2$. It is optimal when either resource is chosen by one player, yielding $\operatorname{cost}(O P T)=$ $1+2=3$. However, in a subgame perfect equilibrium, both players chose resource 1 , yielding $\operatorname{cost}(S P E)=2+2=4$. We obtain $S P o A \geq 4 / 3$.

\begin{tabular}{|c|c||c|c|}
\hline congestion game & \# players & $P o A$ & $S P o A$ \\
\hline \hline general & $n=2$ & $2[5,2]$ & 1.5 \\
general & $n=3$ & $2.5[5,2]$ & $2 \frac{63}{488}$ \\
singleton & $n \geq 3$ & $2.5[4]$ & $\leq n-1$ \\
singleton & $n \rightarrow \infty$ & $2.5[4]$ & $\geq 2 \frac{1}{e}$ \\
singleton \& symmetric & $n \geq 2$ & $4 / 3[7]$ & $4 / 3$ \\
\hline
\end{tabular}

Fig. 6. Results for the $S P O A$ in comparison to the $P o A$.

\section{Conclusion}

We see that for atomic congestion games with two or three players, the sequential price of anarchy is lower than the price of anarchy. We conjecture this to be 
true also for more than three players. We think it is a good guess that $2+1 / e$ is the answer also in general, and as long as an analytic proof is lacking, the conjecture can be backed by more extensive computational experiments (with a finite number of players). Figure 6 gives a brief overview.

\section{References}

1. A. Angelucci, V. Bilo, M. Flammini and L. Moscardelli. On the Sequential Price of Anarchy of Isolation Games, In:Proceedings 19th COCOON, Lecture Notes in Computer Science, Vol. 7936, 17-28, 2013

2. B. Awerbuch, Y. Azar, and A. Epstein. The Price of Routing Unsplittable Flow, In:Proceedings 37th STOC, 57-66, 2005.

3. V. Bilo, M. Flammini, G. Monaco, and L. Moscardelli. Some Anomalies of Farsighted Strategic Behavior, In:Proceedings 10th WAOA, Lecture Notes in Computer Science, Vol. 7846, 229-241, 2013

4. I. Caragiannis, M. Flammini, C. Kaklamanis, P. Kanellopoulos, and L. Moscardelli. Tight Bounds for Selfish and Greedy Load Balancing, In:Proceedings 33rd ICALP, Lecture Notes in Computer Science, Vol. 4051, 311-322, 2006.

5. G. Christodoulou, and E. Koutsoupias. The Price of Anarchy of Finite Congestion Games, In:Proceedings 37th STOC, 67-73, 2005.

6. J. de Jong, M. Uetz and A. Wombacher. Decentralized Throughput Scheduling, In:Proceedings 8th CIAC, Lecture Notes in Computer Science, Vol. 7878, 134-145, 2013

7. D. Fotakis. Stackelberg Strategies for Atomic Congestion Games, Theory of Computing Systems, Vol. 47, 218-249, 2010

8. E. Koutsoupias and C. Papadimitriou. Worst-case equilibria. In:Proceedings 16th STACS, Lecture Notes in Computer Science 1563, 404-413. Springer, 1999.

9. H. W. Kuhn. Extensive Games and the Problem of Information, Contribution to the Theory of Games, Vol. II, Annals of Math. Studies, 28, 193-216, 1953.

10. T. Lücking, M. Mavronicolas, B. Monien, and M. Rode. A New Model for Selfish Routing, In:Proceedings STACS 2004, Lecture Notes in Computer Science, Vol. 2996, 547-558, 2004

11. R. Paes Leme, V. Syrgkanis, and É Tardos. The Curse of Simultaneity. In: Proceedings 3rd ITCS, 60-67, ACM, 2012.

12. R. W. Rosenthal. A class of games possessing pure-strategy Nash equilibria. International Journal of Game Theory, Vol. 2, 65-67, 1973.

13. T. Roughgarden. Selfish routing with atomic players, In:Proceedings 16th SODA, 1184-1185, 2005.

14. R. Selten. A simple model of imperfect competition, where 4 are few and 6 are many, International Journal of Game Theory, Vol. 2, 141-201, Physica Verlag 1973.

15. S. Suri, C. Toth, and Y. Zhou, Selfish Load Balancing and Atomic Congestion Games, Algorithmica, Vol. 47, 79-96, 2007.

\section{A ILP Formulation for 3 players}

Here we give the ILP formulation we use in section 5 . In the formulation, we de-

note by $\mathcal{A}$ the set of all actions $\bigcup_{i \in N} \mathcal{A}_{i}$. The ILP uses $2^{2+3+7}=4096$ resources $R$, one for every combination of actions. Note that here we denote actions by 
lowercase letters $a, b$, and $c$, because they appear as index to the decision variables. We use binary parameters $\delta_{a r}$ to specify whether resource $r$ is chosen in action $a$. For each resource $r$, we have decision variables $d_{r}$ and $w_{r}$, the constant cost and weight of $r$, respectively.

We denote by $v_{a}=\sum_{r \in R} \delta_{a r}\left(w_{r}+d_{r}\right)$ the cost of a player that chooses action $a$ without taking any other players' actions into consideration. Next, we denote by $o_{a b}=\sum_{r \in R} \delta_{a r} \delta_{b r} w_{r}$ the additional costs that two players with actions $a$ and $b$ incur due to overlap in resources. We use these auxiliary variables to determine the total cost of player $i$, for $i \in\{1,2,3\}$, when players 1,2 and 3 choose actions $a, b$ and $c$, respectively. This we denote by $\operatorname{cost}_{i}(a, b, c)$.

We define the outcome where each player chooses his first action $(1,1,1)$ as the social optimum with total costs normalized to 1 . We use binary variables $x_{a}^{1}, x_{a b}^{2}$ and $x_{a b c}^{3}$ to determine which actions are subgame perfect. For example, $x_{a b}^{2}=0$ whenever action $b$ is subgame perfect for player 2 , anticipating a subgame perfect action of player 3 , and knowing that player 1 has chosen action $a$, and $x_{a b}^{2}=1$ otherwise.

Finally, $\operatorname{cost}_{1}(a)$ and $\operatorname{cost}_{2}(a b)$ determine the cost of actions of players 1 and 2 respectively, when successors play subgame perfect. For instance, $\operatorname{cost}_{2}(a b)$ denotes the cost of action $b$ for player 2, when player 1 chooses action $a$, and player 3 plays subgame perfect, given actions $a$ and $b$ of players 1 and 2 , respectively. Finally cost ${ }^{S P E}$ determines the sum of costs for all players in the outcome corresponding to the subgame perfect equilibrium.

\section{Parameters}

$$
\delta_{a r} \forall r \in R, \forall a \in \mathcal{A} \begin{cases}0 & \text { if } r \in A \\ 1 & \text { otherwise }\end{cases}
$$

\section{Variables}

$$
\begin{array}{ll}
w_{r} \forall r \in R & \text { the weight of resource } r \\
d_{r} \forall r \in R & \text { the constant cost of resource } r \\
v_{a} \forall a \in \mathcal{A} & \text { the total constant cost plus weight of all resources in } a \\
o_{a b} \forall a \in \mathcal{A}, b \in \mathcal{A}, a \neq b \text { the total weight of resources that are in } a \text { and } b \\
\operatorname{cost}_{i}(a b c) \forall a \in \mathcal{A}_{1}, b \in \mathcal{A}_{2}, c \in \mathcal{A}_{3}, i \in N \text { the costs of player } i \text { when players } \\
1,2,3 \text { choose } a, b, c \text { respectively }
\end{array}
$$

$\operatorname{cost}^{S P E}$

$$
\operatorname{cost}_{1}(a) \quad \forall a \in \mathcal{A}_{1}
$$$$
\operatorname{cost}_{2}(a b) \quad \forall a \in \mathcal{A}_{1}, \forall b \in \mathcal{A}_{2}
$$

the total costs in the $S P E$.

the costs of player 1 when he plays $a$ and 2,3 play subgame perfect

the costs of player 2 when players 1,2 play $a, b$ respectively and 3 plays subgame perfect 


$$
\begin{aligned}
& x_{a}^{1} \quad \forall a \in \mathcal{A}_{1} \\
& x_{a b}^{2} \forall a \in \mathcal{A}_{1}, b \in \mathcal{A}_{2} \\
& x_{a b c}^{3} \forall a \in \mathcal{A}_{1}, b \in \mathcal{A}_{2}, c \in \mathcal{A}_{3} \begin{cases}0 & \text { if } a \text { is subgame perfect for player } 1 \\
1 & \text { otherwise } \\
0 & \text { if } a b \text { is subgame perfect for player } 2 \\
1 & \text { otherwise } \\
0 & \text { if } a b c \text { is subgame perfect for player } 3 \\
1 & \text { otherwise }\end{cases}
\end{aligned}
$$

\section{Constraints}

$$
\begin{aligned}
& v_{a}=\sum_{r \in R} \delta_{a r}\left(w_{r}+d_{r}\right) \quad \forall a \in \mathcal{A} \\
& o_{a b}=\sum_{r \in R} \delta_{a r} \delta_{b r} w_{r} \forall a \in \mathcal{A}, \forall b \in \mathcal{A}, a \neq b \\
& \operatorname{cost}_{1}(a b c)=v_{a}+o_{a b}+o_{a c} \quad \forall a \in \mathcal{A}_{1}, b \in \mathcal{A}_{2}, c \in \mathcal{A}_{3} \\
& \operatorname{cost}_{2}(a b c)=v_{b}+o_{a b}+o_{b c} \quad \forall a \in \mathcal{A}_{1}, b \in \mathcal{A}_{2}, c \in \mathcal{A}_{3} \\
& \operatorname{cost}_{3}(a b c)=v_{c}+o_{a c}+o_{b c} \quad \forall a \in \mathcal{A}_{1}, b \in \mathcal{A}_{2}, c \in \mathcal{A}_{3} \\
& \operatorname{cost}_{1}(111)+\operatorname{cost}_{2}(111)+\operatorname{cost}_{3}(111)=1 \\
& \operatorname{cost}_{1}(a b c)+\operatorname{cost}_{2}(a b c)+\operatorname{cost}_{3}(a b c) \geq 1 \forall a \in \mathcal{A}_{1}, b \in \mathcal{A}_{2}, c \in \mathcal{A}_{3} \\
& \sum_{a \in \mathcal{A}_{1}} x_{a}^{1} \leq\left|\mathcal{A}_{1}\right|-1 \\
& \sum_{b \in \mathcal{A}_{2}} x_{a b}^{2} \leq\left|\mathcal{A}_{2}\right|-1 \quad \forall a \in \mathcal{A}_{1} \\
& \sum_{c \in \mathcal{A}_{3}} x_{a b c}^{3} \leq\left|\mathcal{A}_{3}\right|-1 \forall a \in \mathcal{A}_{1}, \forall b \in \mathcal{A}_{2} \\
& \operatorname{cost}_{3}(a b c) \leq \operatorname{cost}_{3}\left(a b c^{\prime}\right)+M \cdot x_{a b c}^{3} \forall a \in \mathcal{A}_{1}, b \in \mathcal{A}_{2}, c \in \mathcal{A}_{3}, c^{\prime} \in \mathcal{A}_{3} \\
& \operatorname{cost}_{2}(a b) \leq \operatorname{cost}_{2}\left(a b^{\prime}\right)+M \cdot x_{a b}^{2} \quad \forall a \in \mathcal{A}_{1}, b \in \mathcal{A}_{2}, b^{\prime} \in \mathcal{A}_{2} \\
& \operatorname{cost}_{1}(a) \leq \operatorname{cost}_{1}\left(a^{\prime}\right)+M \cdot x_{a}^{1} \quad \forall a \in \mathcal{A}_{1}, a^{\prime} \in \mathcal{A}_{1} \\
& \operatorname{cost}_{1}(a) \leq \operatorname{cost}_{1}(a b c)+M \cdot\left(x_{a b}^{2}+x_{a b c}^{3}\right) \forall a \in \mathcal{A}_{1}, b \in \mathcal{A}_{2}, c \in \mathcal{A}_{3} \\
& \operatorname{cost}_{1}(a) \geq \operatorname{cost}_{1}(a b c)-M \cdot\left(x_{a b}^{2}+x_{a b c}^{3}\right) \forall a \in \mathcal{A}_{1}, b \in \mathcal{A}_{2}, c \in \mathcal{A}_{3} \\
& \operatorname{cost}_{2}(a b) \leq \operatorname{cost}_{2}(a b c)+M \cdot x_{a b c}^{3} \forall a \in \mathcal{A}_{1}, b \in \mathcal{A}_{2}, c \in \mathcal{A}_{3} \\
& \operatorname{cost}_{2}(a b) \geq \operatorname{cost}_{2}(a b c)-M \cdot x_{a b c}^{3} \forall a \in \mathcal{A}_{1}, b \in \mathcal{A}_{2}, c \in \mathcal{A}_{3}
\end{aligned}
$$




$$
\begin{aligned}
\operatorname{cost}^{S P E} \leq \operatorname{cost}_{1}(a b c)+\operatorname{cost}_{2}(a b c)+\operatorname{cost}_{3}(a b c) & +M \cdot\left(x_{a}^{1}+x_{a b}^{2}+x_{a b c}^{3}\right) \\
& \forall a \in \mathcal{A}_{1}, b \in \mathcal{A}_{2}, c \in \mathcal{A}_{3}
\end{aligned}
$$

Constraints (3) and (4) define $v_{a}$ and $o_{a b}$ for all actions $a, b$. Constraints (6),(7), and (8) define the costs in each outcome for each player. Without loss of generality, we normalize the costs such that the optimal solution is the outcome $(1,1,1)$ and has total cost equal to 1 in constraints (9) and (10). Constraints $(11),(12)$, and (13) make sure that there exists at least one subgame perfect action for each player. Constraints (14),(15), and (16) make sure no player can improve from any subgame perfect action; when action $c$ is subgame perfect when players 1,2 choose actions $a, b$ respectively, then $\operatorname{cost}_{3}(a b c) \leq \operatorname{cost}_{3}\left(a b c^{\prime}\right)$ for any action $c^{\prime}$. When action $b$ is subgame perfect for player 2 when player 1 chooses action $a$, then $\operatorname{cost}_{2}(a b) \leq \operatorname{cost}_{2}\left(a b^{\prime}\right)$ for all actions $b^{\prime}$. When action $a$ is subgame perfect for player 1 , then $\operatorname{cost}_{1}(a) \leq \operatorname{cost}_{1}\left(a^{\prime}\right)$ for all actions $a^{\prime}$. Constraints (17) and (18) define $\operatorname{cost}_{1}(i)$; if actions $b$ and $c$ are subgame perfect for players 2,3 respectively, when player 1 chooses action $a$, then $\operatorname{cost}_{1}(a)=\operatorname{cost}_{1}(a b c)$. Constraints (17) and (18) define $\operatorname{cost}_{2}(a b)$; if $c$ is subgame perfect for player 3, when players 1,2 chooses actions $a, b$ respectively, then $\operatorname{cost}_{2}(a b)=\operatorname{cost}_{2}(a b c)$. Constraint (21) defines cost ${ }^{S P E}$; if actions $a, b, c$ are subgame perfect for players $1,2,3$ respectively, then $\operatorname{cost}^{S P E}=\operatorname{cost}_{1}(a b c)+\operatorname{cost}_{2}(a b c)+\operatorname{cost}_{3}(a b c)$. Since we maximize cost ${ }^{S P E}$, an inequality suffices. Constraints (14) to (20), are 'big M' constraints; if any binary variable equals 1 (in which case the corresponding action is not subgame perfect), then the constraint does not impose any restriction. Due to lemma $5, M=n$ suffices.

\section{Objective}

The objective is to maximize $\operatorname{cost}^{S P E}$, since, due to the normalization, this value equals the sequential price of anarchy.

\section{B Lower Bound Example for 3 players}

Here we give the lower bound example that we use in Section 5, scaled to integers such that $\operatorname{cost}(O P T)=488$.

Example 4. There are 13 resources $\{1, \ldots, 13\}$ with weights $w_{1}=84, w_{2}=$ $52, w_{3}=3, w_{4}=4, w_{5}=31, w_{6}=52, w_{7}=54, w_{8}=92, w_{9}=51, w_{10}=$ $28, w_{11}=4, w_{12}=33, w_{13}=374$. Player 1 has 2 actions $\{1.1,1.2\}$. Player 2 has 3 actions $\{2.1,2.2,2.3\}$. Player 3 has 4 actions $\{3.1,3.2,3.3,3.4\}$. Table 1 shows for each action, which resources it contains. This example is shown in Figure 7.

Calculating the costs of all actions in the game tree yields a $S P o A$ where player 1 chooses 1.2 , player 2 chooses 2.3 , and player 3 chooses 3.4 , yielding a

total cost of $C(S P O A)=1039$. Therefore $S P O A=\frac{1039}{488}=2 \frac{63}{488}$. The game tree is shown in Figure 8. 


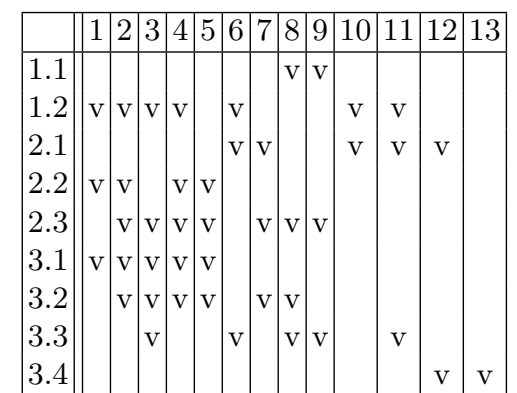

Table 1. v denotes that the corresponding action contains the corresponding resource

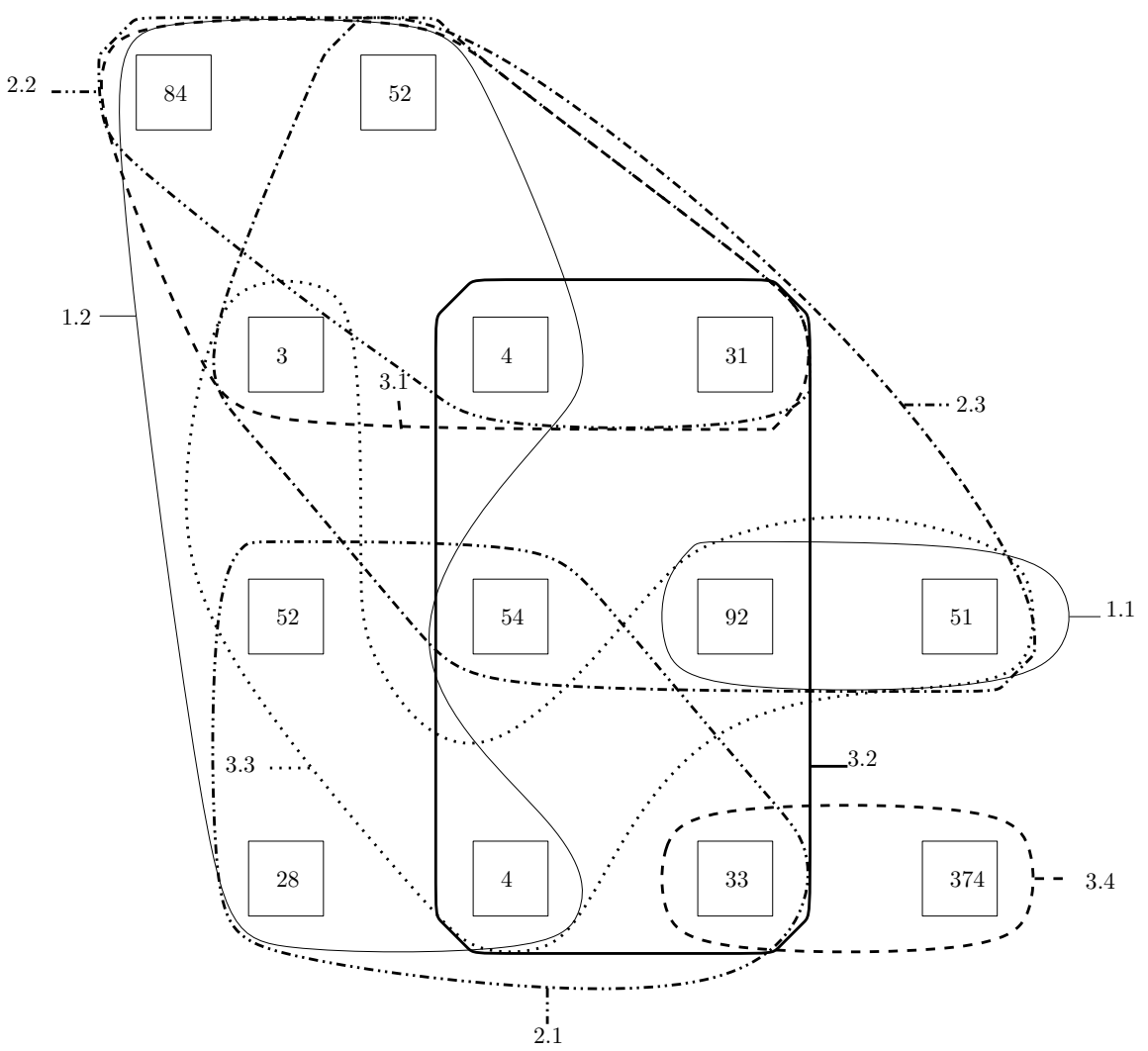

Fig. 7. The lower bound example for 3 players. Squares correspond to resources. The number in each resource denotes its weight. Encircled areas correspond to actions. 


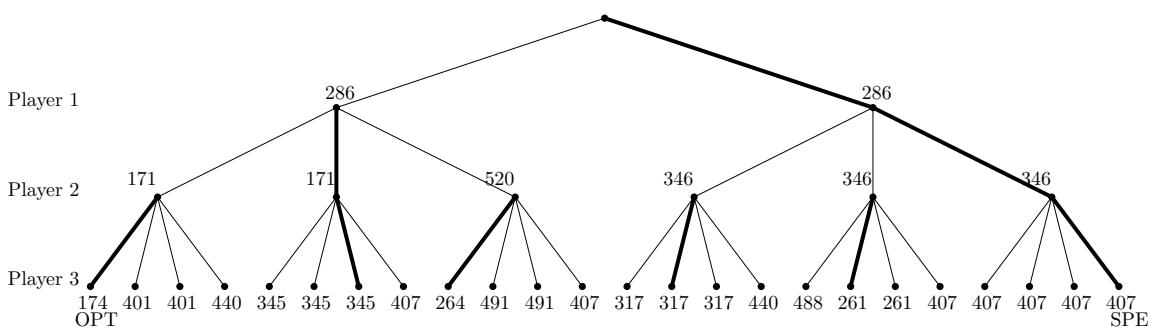

Fig. 8. The game tree for three players. The number at each action denotes the cost of the corresponding player when all successors play subgame perfect. Fat lines correspond to subgame perfect actions.

\section{An Upper Bound For Singleton Congestion Games with $n>3$ Players}

Here we prove the upper bound $n-1$ for singleton congestion games. We start with the following lemma.

Lemma 7. Suppose players $1, \ldots, i$ choose resources $r_{1}, \ldots, r_{i}$, and suppose it is subgame perfect for every player $j$ succeeding $i$ to choose $r_{i}$. Then it is also subgame perfect for every player $j$ succeeding $i$ to choose $r_{i}$, when players $1, \ldots, i$ choose $r_{1}, \ldots r_{i-1}, r_{i}^{\prime}$ instead.

Proof. Denote by $A$ the outcome when players $1 \ldots i$ choose resources $r_{1}, \ldots, r_{i}$, and every player $j$ succeeding $i$ chooses $r_{i}$. Suppose the lemma is false, and suppose players $1 \ldots i$ choose $r_{1}, \ldots, r_{i-1}, r_{i}^{\prime}$. Then, for any subgame perfect equilibrium for players $i+1, \ldots, n$, there exists a last player $j$ that chooses some resource $r_{j} \neq r_{i}$. Now compared to $A$, player $i$ does not choose $r_{i}$, no additional players choose $r_{i}$, and resources other than $r_{i}$ are chosen at least as often. Therefore, for player $j, r_{i}$ costs less than in $A$, while other resources cost as least as much as in $A$, no matter what the players succeeding $j$ will do. In $A$, however, choosing $r_{i}$ was subgame perfect. Therefore, it cannot be subgame perfect for $j$ to choose $r_{j}$ instead of $r_{i}$. This is a contradiction.

Using this lemma, we lower bound the utility of players in the optimum in the following lemma.

Lemma 8. Consider an instance where there exists a player $i$, such that it is subgame perfect for every player preceding $i$ to choose $r_{i}^{*}$, and if players $1 \ldots i$ choose resources $r_{i}^{*}, \ldots, r_{i}^{*}$, then it is subgame perfect for every player succeeding $i$ to choose $r_{i}^{*}$. Then for any player $k \neq i$ either $i$ chooses $r_{k}^{*}$ in SPE, or we have $d_{r_{k}^{*}}+w_{r_{k}^{*}} \geq d_{r_{i}^{*}}+(n-1) w_{r_{i}^{*}}$. Here $r_{i}^{*}$ and $r_{k}^{*}$ denote the resources players $i$ and $k$ choose in the optimum solution, respectively.

Proof. Suppose there exists such a player $i$. Due to Lemma 7, there exists a subgame perfect equilibrium $S P E$, where each player other than $i$ chooses $r_{i}^{*}$ 
(and $i$ chooses some resource, which is either $r_{i}^{*}$ or some other resource). Consider resource $r_{k}^{*}$ for some player $k$. Denote the set of players that can choose $r_{k}^{*}$ by $N^{\prime}$. Since $k$ can choose $r_{k}^{*}, N^{\prime}$ is not empty, therefore it has a last player $j$. Now, suppose in $S P E$, player $i$ does not choose $r_{k}^{*}$. Then player $j$ can choose $r_{k}^{*}$, obtaining cost $d_{r_{k}^{*}}+w_{r_{k}^{*}}$. Since he obtains a cost of $d_{r_{k}^{*}}+w_{r_{k}^{*}}$ instead, we see that $d_{r_{k}^{*}}+w_{r_{k}^{*}} \geq d_{r_{i}^{*}}+(n-1) w_{r_{i}^{*}}$, proving the lemma.

Now we are ready to prove the theorem.

Theorem 4. For singleton atomic congestion games with affine cost functions, $S P O A \leq n-1$.

Proof. Denote by $r_{i}^{*}$ the resource player $i$ chooses in the optimum solution. Suppose $S P o A>n-1$. Then there exists at least one player $i$ with $\operatorname{cost}_{i}(S P E)>$ $(n-1) \operatorname{cost}_{i}(O P T) \geq(n-1)\left(d_{r_{i}^{*}}+w_{r_{i}^{*}}\right) \geq d_{r_{i}^{*}}+(n-1) w_{r_{i}^{*}}$. Therefore in SPE, each player $j$ preceding $i$ chooses $r_{i}^{*}$, and, if player $i$ chooses $r_{i}^{*}$, it is subgame perfect for each player $k$ succeeding $i$ to choose $r_{i}^{*}$. If in SPE player $i$ chooses $r_{i}^{*}$, then $\operatorname{cost}(S P E)=n d_{r_{i}^{*}}+n^{2} w_{r_{i}^{*}}$. If player $i$ chooses another resource, then player $i$ does not incur a higher cost, while all other players incur a smaller cost. Therefore $\operatorname{cost}(S P E) \leq n d_{r_{i}^{*}}+n^{2} w_{r_{i}^{*}}$.

Since $\operatorname{cost}_{i}(S P E)>d_{r_{i}^{*}}+(n-1) w_{r_{i}^{*}}$, due to lemma 8, we have $d\left(r_{k}^{*}\right)+w\left(r_{k}^{*}\right) \geq$ $d_{r_{i}^{*}}+(n-1) w_{r_{i}^{*}} \forall k \in N$. Therefore $\operatorname{cost}(O P T)=\sum_{j \neq i} \operatorname{cost}_{j}(O P T)+\operatorname{cost}_{i}(O P T) \geq$ $\sum_{j \neq i}\left(d_{r_{i}^{*}}+(n-1) w_{r_{i}^{*}}\right)+d_{r_{i}^{*}}+w_{r_{i}^{*}}=n d_{r_{i}^{*}}+\left((n-1)^{2}+1\right) w_{r_{i}^{*}}$. Finally

$S P O A \leq \frac{\operatorname{cost}(S P E)}{\operatorname{cost}(O P T)} \leq \frac{n d_{r_{i}^{*}}+n^{2} w_{r_{i}^{*}}}{n d_{r_{i}^{*}}+\left((n-2)^{2}+1\right) w_{r_{i}^{*}}} \leq n-1$ for $n \geq 3$. This contradicts our assumption. Therefore $S P O A \leq n-1$ for $n \geq 3$. 